\title{
Nanoantenna-assisted plasmonic enhancement of IR absorption of vibrational modes of organic molecules
}

\author{
Alexander G. Milekhin ${ }^{* 1,2}$, Olga Cherkasova ${ }^{2,3}$, Sergei A. Kuznetsov ${ }^{2,4}$, \\ Ilya A. Milekhin ${ }^{1,2}$, Ekatherina E. Rodyakina ${ }^{1,2}$, Alexander V. Latyshev ${ }^{1,2}$, \\ Sreetama Banerjee ${ }^{5}$, Georgeta Salvan ${ }^{5}$ and Dietrich R. T. Zahn ${ }^{5}$
}

\section{Full Research Paper}

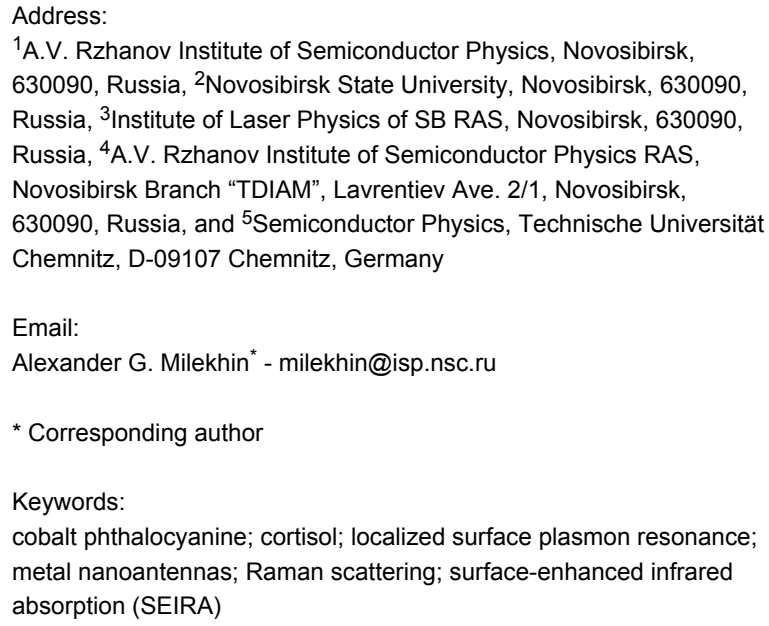

${ }^{1}$ A.V. Rzhanov Institute of Semiconductor Physics, Novosibirsk, 630090, Russia, ${ }^{2}$ Novosibirsk State University, Novosibirsk, 630090, Russia, ${ }^{3}$ Institute of Laser Physics of SB RAS, Novosibirsk, 630090, Russia, ${ }^{4}$ A.V. Rzhanov Institute of Semiconductor Physics RAS, Novosibirsk Branch “TDIAM", Lavrentiev Ave. 2/1, Novosibirsk, 630090, Russia, and ${ }^{5}$ Semiconductor Physics, Technische Universität Chemnitz, D-09107 Chemnitz, Germany

Email:

Alexander G. Milekhin* - milekhin@isp.nsc.ru

* Corresponding author

Keywords:

cobalt phthalocyanine; cortisol; localized surface plasmon resonance; metal nanoantennas; Raman scattering; surface-enhanced infrared absorption (SEIRA)

Beilstein J. Nanotechnol. 2017, 8, 975-981. doi:10.3762/bjnano.8.99

Received: 24 November 2016

Accepted: 07 April 2017

Published: 03 May 2017

This article is part of the Thematic Series "Towards molecular spintronics".

Associate Editor: P. Leiderer

(C) 2017 Milekhin et al.; licensee Beilstein-Institut. License and terms: see end of document.

\begin{abstract}
Nanoantenna-assisted plasmonic enhancement of IR absorption and Raman scattering was employed for studying the vibrational modes in organic molecules. Ultrathin cobalt phthalocyanine films (3 nm) were deposited on Au nanoantenna arrays with specified structural parameters. The deposited organic films reveal the enhancement of both Raman scattering and IR absorption vibrational modes. To extend the possibility of implementing surface-enhanced infrared absorption (SEIRA) for biological applications, the detection and analysis of the steroid hormone cortisol was demonstrated.
\end{abstract}

\section{Introduction}

Organic semiconductors have been extensively investigated during the past few decades due to their wide range of applications in various organic-inorganic hybrid devices [1]. The ability to tailor the chemical structure of the organic molecules according to the device requirements, in addition to the light weight, flexibility and easy processing of these materials, open up the possibility of fabricating novel hybrid devices [2]. In the last decade, organic semiconductors gained the attention of the spintronics community as these organic semiconductors have been considered as good candidates for spin transport. The most interesting property of organic semiconductors for spintronic applications is the weak spin-scattering mechanism [3], which means that the spin polarization of the carriers can continue for an extended time (in the range from microseconds to millisec- 
onds) [4]. This feature is caused by very low spin-orbit coupling and weak hyperfine interaction.

Phthalocyanines (Pcs) are a class of stable, planar small molecules, often investigated as promising candidates for molecular spintronics $[5,6]$. There have been studies on molecule/metal interfaces [7], magnetic coupling in the metal phthalocyanine layers [8], spin transport or magnetic properties through single molecules or even thin layers $[6,9,10]$ of phthalocyanines These molecules also offer the possibility of changing the spindependent transport mechanism by slightly modifying the molecular structure [6]. It has been previously shown that the molecular structure of a magnetic material can be probed by various spectroscopic techniques [11]. Under conditions of plasmonic enhancement, a magnetic material can be employed for a wide range of applications [11].

A relatively low optical signal from the vibrational modes of organic molecules using conventional spectroscopic techniques such as infrared (IR) and Raman spectroscopy restricts their detection limit, which is crucial for sensor applications. The sensitivity of these optical methods can be drastically increased by implementation of nanoantenna-assisted plasmonic-enhanced spectroscopy techniques such as surface-enhanced IR absorption (SEIRA) [12] or surfaced-enhanced Raman scattering (SERS) [13]. The principle of SEIRA and SERS is based on specially designed, resonant, metal nanoantennas, providing a high electromagnetic field intensity in close proximity to the plasmonic nanostructure when resonantly excited in the IR or optical regime $[14,15]$. It was shown that elongated nanoantennas can enhance the SEIRA signal by molecular vibrations in model adsorbates such as octadecanthiol (ODT) [16] and 4,4'bis( $N$-carbazolyl)-1,1'-biphenyl (CBP) by up to five orders of magnitude [17]. The IR absorption bands of these molecules become pronounced, even for molecular monolayers, by tuning the localized surface plasmon energy of the nanoantennas to the energy of the molecular vibrations. Along with SEIRA, SERS is also traditionally used to study the vibrational spectra of various organic and biological substances [18], which may be present in very low quantities down to single molecules [19]. Raman enhancement up to $10^{14}$ can be achieved.

Although SEIRA is a relatively new tool for detection of organic and biological substances, it is found to be very effective for probing extremely low concentrations. Adato et al. demonstrated detection of $3 \times 10^{-19}$ moles of silk protein for the entire nanoantenna array, corresponding to only 145 molecules per antenna [20]. A similar approach is used for the terahertz (or far-IR) spectral range for which special nanoscale slotantenna arrays were designed to determine glucose and fructose in solutions, including market beverages [21]. Terahertz transmittance measurements were made in the frequency range of $0.5-2.5 \mathrm{THz}$ for concentrations from 10 to $4168 \mathrm{mg} / \mathrm{dL}$. Similar structures have been used for the determination of pesticide concentration in solution and on the surface of fruit. The detection limit was $8 \mu \mathrm{mol}$ [22].

Here, we demonstrate the application of SEIRA for the detection and analysis of vibrational modes of cobalt phthalocyanine deposited on Au nanoantenna arrays. The estimation of the plasmonic enhancement of the fabricated nanoantenna arrays was performed by analyzing the SEIRA and SERS spectra of homogeneous, ultrathin Co Pc films. We also demonstrate SEIRA by detection of the steroid hormone cortisol deposited on $\mathrm{Au}$ nanoantenna arrays to extend the possibility of using the method also for biological applications.

\section{Results and Discussion Applications of SEIRA for organic compounds}

Representative SEM images of the Au nanoantenna arrays (length $900 \mathrm{~nm}$; width $60 \mathrm{~nm}$ ) employed for the IR investigation are shown in Figure 1. The period of the array is $5 \mu \mathrm{m}$ and the distance between nanoantenna edges is about $100 \mathrm{~nm}$.

For the quantitative estimation of the nanoantenna-assisted plasmonic enhancement of the fabricated arrays, ultrathin $\mathrm{CoPc}$ films (with a thickness up to $3 \mathrm{~nm}$ ) were deposited on the

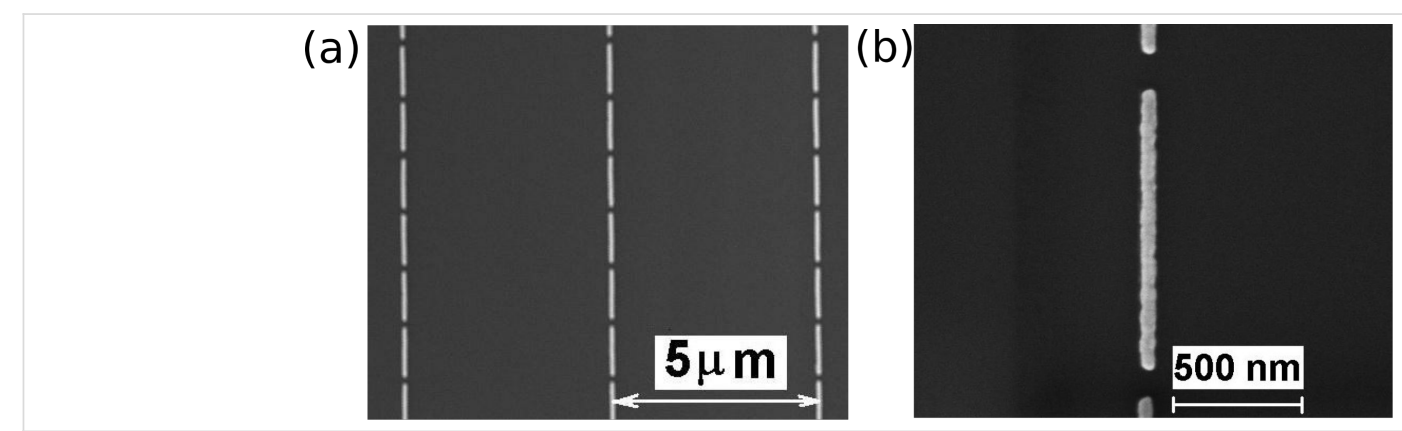

Figure 1: (a) Representative SEM image of Au nanoantenna array; nanoantenna length $900 \mathrm{~nm}$. (b) Detailed image of a nanoantenna. 
antenna arrays and a Si substrate. The homogeneity of the CoPc films deposited on the arrays was probed using micro-Raman mapping. The Raman spectra of the CoPc films deposited on a Si substrate (Figure 2a) reveal a rich spectrum of CoPc vibrational modes similar to that observed previously [23]. Note that the excitation energy was $1.96 \mathrm{eV}(632.8 \mathrm{~nm})$, which matches well with the HOMO-LUMO gap energy of CoPc $(1.9 \mathrm{eV})$. The coincidence of the excitation energy with that of the electronic transitions in CoPc defines the conditions for resonant Raman scattering (RRS) in CoPc. In addition to the most intense mode of the Si substrate (observed at $520.5 \mathrm{~cm}^{-1}$ ), the RRS spectrum (Figure 2) is dominated by the vibrational mode at $1543 \mathrm{~cm}^{-1}$ assigned to the $\mathrm{C}=\mathrm{N}$ stretching mode [23]. The chemical structure of $\mathrm{CoPc}$ is shown in the inset of Figure 2a. The mode frequencies observed at 683, 750, 958, 1307,1340 , and $1465 \mathrm{~cm}^{-1}$ are in accordance with the literature data [23].

The Raman spectra measured from CoPc deposited on the $\mathrm{Au}$ nanoantennas demonstrate the enhancement of the Raman scattering (a factor of about 9) without noticeable shift of the mode frequencies, manifesting a SERS effect by CoPc. In the case of nanoantennas, the SERS enhancement is much weaker than that determined for $\mathrm{CoPc}$ on $\mathrm{Au}$ nanocluster arrays (enhancement factor of $2 \times 10^{4}$ ) observed in our previous experiments [24]. Much stronger SERS enhancement of CoPc on Au nanocluster arrays with respect to that for nanoantennas can be explained by the resonant SERS effect, as the energy of the local surface plasmon resonance (LSPR) of nanoclusters is located in the red spectral region, which is in resonance with the excitation energy. In the case of nanoantennas, the energy of longitudinal LSPR modes polarized along the antennas shifts in the IR spectral range, which leads to off-resonant SERS conditions. The transverse LSPR modes polarized perpendicular to the nanoantennas undergo a small blue shift along with a decrease in the mode intensity [25], which leads to reduced SERS enhancement. Despite this, the SERS effect in CoPc on nanoantenna arrays allows the homogeneity of the CoPc coverage on a nanoantenna array to be investigated using Raman mapping. The intensity of the $\mathrm{C}=\mathrm{N}$ stretching mode at $1543 \mathrm{~cm}^{-1}$ was monitored. The Raman map obtained for a Au nanoantenna array with a $3 \mathrm{~nm}$ thick CoPc film shown in Figure 2b,c agrees well with the SEM image of the same structure. One can see from Figure $2 \mathrm{c}$ that the Raman mapping indicates the position of the nanoantennas by the stronger Raman (SERS) intensity (brighter regions), which reproduces the $5 \mu \mathrm{m}$ periodicity of the
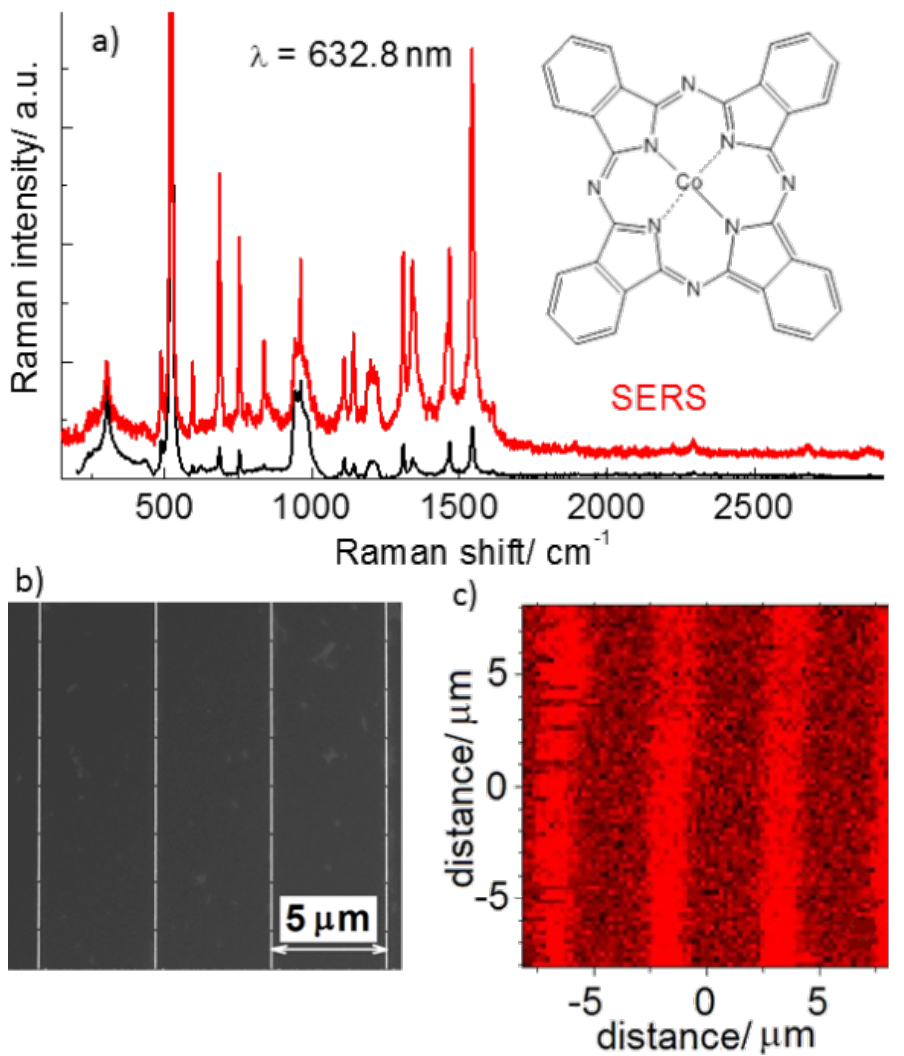

Figure 2: (a) Raman and SERS spectra of a cobalt phthalocyanine (CoPc) film with thickness of 3 nm deposited on a nanoantenna array. The chemical structure of CoPc is shown in the inset. (b) SEM image of the nanoantenna array with antenna length of $1900 \mathrm{~nm}$. (c) Raman mapping $(100 \times 100$ pixels $)$ of the $\mathrm{C}=\mathrm{N}$ mode $\left(1543 \mathrm{~cm}^{-1}\right)$ intensity. 
nanoantenna array and evidences the homogeneous coverage of CoPc over the sample.

The IR spectrum of a $10 \mathrm{~nm}$ thick CoPc film is presented in Figure 3 in the spectral range of $500-2000 \mathrm{~cm}^{-1}$, where most absorption lines of $\mathrm{CoPc}$ are located. The vibrational spectrum of the CoPc film is similar to that defined for CoPc earlier [2631] by means of IR absorption. The absorption line at $726 \mathrm{~cm}^{-1}$ attributed to nonplanar deformation of $\mathrm{C}-\mathrm{H}$ bonds of benzene rings [26-28] has the strongest intensity and, therefore, was chosen for further SEIRA experiments. It is accompanied by a weaker mode at $755 \mathrm{~cm}^{-1}$, referred to as the nonplanar (out-ofplane) bending of $\mathrm{C}-\mathrm{H}$ bonds and the $\mathrm{Co}-\mathrm{N}$ bond vibrations [26-28].

Au nanoantenna arrays with structural parameters (nanoantenna length and period) designed to ensure the LSPR band energy from 600 to $1000 \mathrm{~cm}^{-1}$ were fabricated (Figure 4a). In

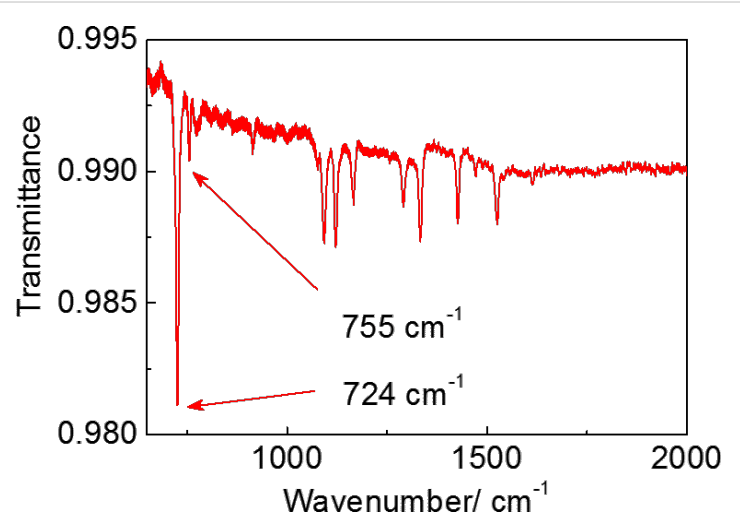

Figure 3: IR transmission spectrum of a $10 \mathrm{~nm}$ thick CoPc film deposited on a Si substrate normalized to the IR spectrum of a Si substrate. order to determine the structural parameters of the arrays with the targeted LSPR energy and a maximal SEIRA enhancement, 3D full-wave simulations were carried out and the distribution of the electromagnetic field near Au nanoantennas on a silicon substrate was simulated as described in [32]. In the simulations, the same nominal values of nanoantenna width, height, and the spacing between nanoantennas were assumed as imposed by the nanofabrication technology.

Except for the fundamental LSPR mode, the third-order resonance centered at about $2400 \mathrm{~cm}^{-1}$ occurs in the IR spectra. A weaker feature near $1200 \mathrm{~cm}^{-1}$ is assigned to the surface optical mode from natural silicon oxide covering the Si substrate [33]. The deposition of thin CoPc films on the nanoantenna arrays leads to intensity enhancement for the vibrational modes at 724 and $755 \mathrm{~cm}^{-1}$, which are inherent to $\mathrm{CoPc}$ in the spectral range of the LSPR band. Note that for the $3 \mathrm{~nm}$ thick CoPc film, the mode at $755 \mathrm{~cm}^{-1}$ is observed only in the case of the CoPc film deposited on the nanoantenna array (Figure 4a,b). Weak oscillations seen in Figure $4 \mathrm{a}, \mathrm{b}$ are the interference fringes at the sample thickness (about $400 \mu \mathrm{m}$ ). The overall enhancement factor (EF) for the $10 \mathrm{~nm}$ thick CoPc film amounts to 2 and is within the range of $4-5$ for a $3 \mathrm{~nm}$ thick film. The increase of the EF with decreasing CoPc film thickness is due to the strong electric filed localization near the Si surface in the nanogap between the nanoantenna edges. Using the approach similar to that described in [34], one can quantitatively estimate the enhancement induced by nanoantennas $\left(\mathrm{EF}_{\mathrm{N}}\right)$. It can be calculated as the ratio of the areas covered by the nanogaps to the entire nanoantenna array and amounts to about 1/1200. Here, we suppose that the IR absorption predominantly takes place in the nanogap. Taking the maximum $\mathrm{EF}=5$ for the $3 \mathrm{~nm}$ thick $\mathrm{CoPc}$ film, the enhancement induced by nanogaps in the array reaches the value of $\mathrm{EF}_{\mathrm{N}}=6000$.
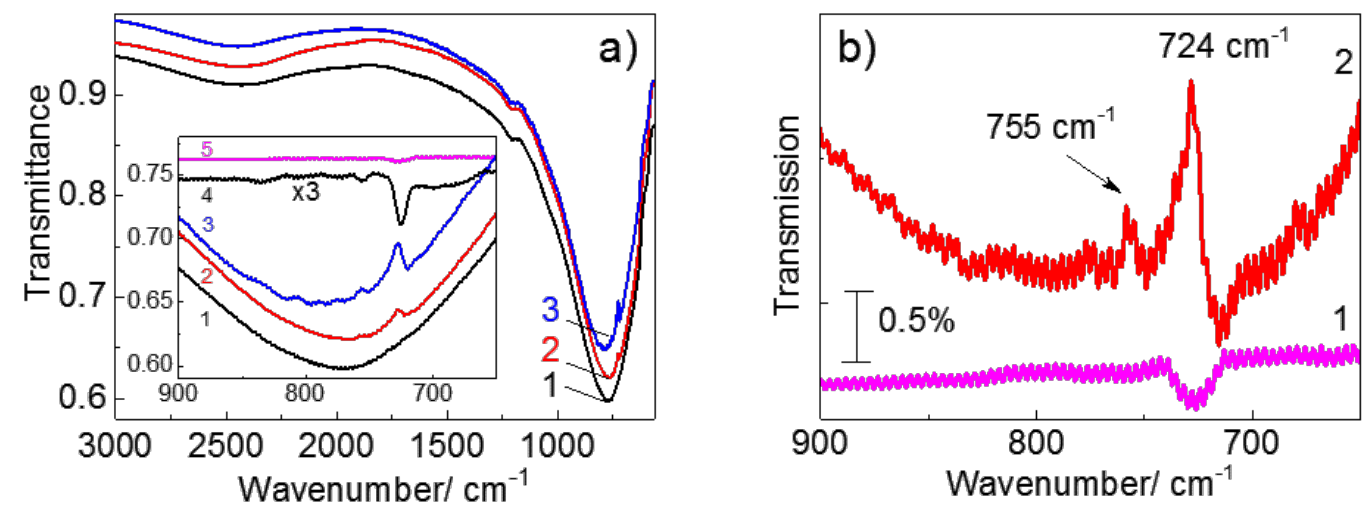

Figure 4: (a) IR spectrum of bare nanoantennas (curve 1) and IR spectra of nanoantennas with deposited $3 \mathrm{~nm}$ and $10 \mathrm{~nm}$ thick CoPc films (curves 2 and 3 , respectively). The inset shows a comparison of the same spectra with the IR spectra of $3 \mathrm{~nm}$ and $10 \mathrm{~nm}$ thick CoPc films on a Si substrate without nanoantennas (curves 4 and 5, respectively). (b) Comparison of IR spectrum of bare nanoantennas (curve 1) and with the spectrum of the structure with the $3 \mathrm{~nm}$ thick CoPc film (curve 2) after background subtraction. 
In accordance with previous studies [18,20,35], the vibrational modes observed in the SEIRA spectra exhibit a clear Fano line shape.

\section{Further applications of SEIRA for biological compounds}

A similar approach was used for the investigation of cortisol using SEIRA. Among other organic compounds, the steroid hormones are of great importance because they influence many physiological processes in humans and animals.

Cortisol is the major glucocorticoid hormone produced in the adrenal gland and in several tissues and regulates blood glucose levels. Cortisol production disorders lead to the development of diabetes, Cushing's syndrome and other pathologies [36]. Therefore, the determination of the cortisol concentration in blood and tissue is important. The immunoassay methods are predominantly performed in clinical practice [37]. Other methods, such as chromatography [38-40] and surface plasmon resonance [41], are used in fundamental research. Each of these methods of cortisol detection has advantages and drawbacks. Conventional Raman scattering, which is widely used for the investigation of organic and inorganic materials, cannot be applied for investigation of cortisol in blood and tissues due to its low Raman signal response. Therefore, SEIRA is considered as a complementary method for enhanced cortisol detection. Cortisol is the derivative of 1,2-cyclopentanephenanthrene. The molecule core of cortisol consists of four fused carbon rings: three cyclohexane rings (denoted as $\mathrm{A}, \mathrm{B}$, and $\mathrm{C}$ in the Figure 5a) and one cyclopentane ring (the $\mathrm{D}$ ring).

The IR spectrum of cortisol reveals the most pronounced absorption bands associated with valence vibrations of $\mathrm{C}=\mathrm{O}$ groups $[42,43]$. According to [44] the calculated (experimental) frequency positions of the bands are located at
$1686(1660) \mathrm{cm}^{-1}$ and $1725(1735) \mathrm{cm}^{-1}$ and demonstrate the highest intensities. Therefore, the structural parameters of the nanoantennas were chosen in such a way to ensure the LSPR absorption band close to the energy of the most pronounced vibrational modes in cortisol. The deposition of cortisol on a solid substrate implies, as a general rule, the use of the dropcasting method, which results in inhomogeneous coverage. The dielectric function of the media surrounding the nanoantennas is changed after deposition of cortisol solution onto the surface. This results in an LSPR energy shift from $1535 \mathrm{~cm}^{-1}$ towards lower energy (near $1500 \mathrm{~cm}^{-1}$ ). Three characteristic vibrational modes of cortisol are also seen in the IR spectrum after subtraction of the envelope line (Figure $5 \mathrm{~b}$ ). These modes can be assigned as deformation vibrations of $\mathrm{C}-\mathrm{H}$ bonds $\left(1470 \mathrm{~cm}^{-1}\right)$, stretching vibrations $\mathrm{C} 3=\mathrm{O} 3\left(1660 \mathrm{~cm}^{-1}\right)$ and $\mathrm{C} 20=\mathrm{O} 20$ bonds $\left(1735 \mathrm{~cm}^{-1}\right)$. The detection limit of the cortisol concentration determined from the IR spectra was $40 \mathrm{ng}$. With increasing cortisol concentration (up to $100 \mathrm{ng}$ ) the intensity of the modes increases and remains unchanged with further concentration increase. The most probable explanation is that the thickness of the cortisol film reaches the value of the nanoantenna height, which prevents further increase of the optical response. The determined detection limit of the cortisol concentration from the analysis of the SEIRA spectra corresponds to the concentration of the steroid in real biological assays.

\section{Conclusion}

In this work, we characterized the nanoantenna-assisted plasmonic enhancement of IR absorption and Raman scattering from vibrational modes of organic molecules. Au nanoantenna arrays with specified structural parameters were employed to enhance the absorption signatures from the vibrational modes of cobalt phthalocyanine ultrathin films and cortisol molecules. This work may have a wide range of applications as it opens up the possibility to spectroscopically study a magnetic material a)

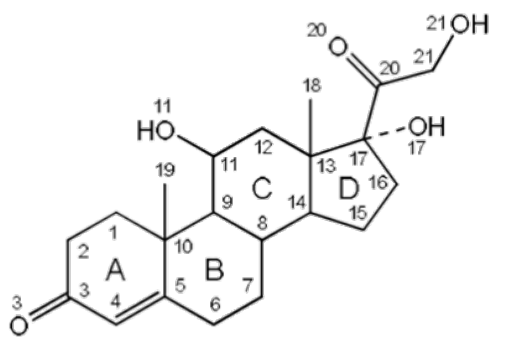

b) 1.0

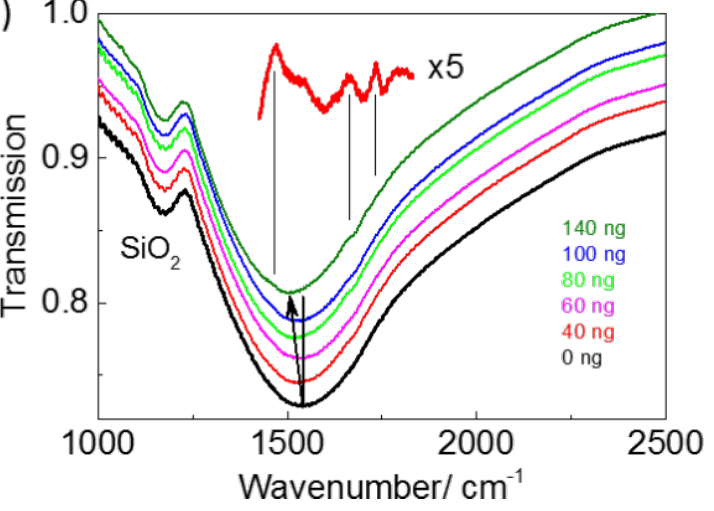

Figure 5: (a) The cortisol chemical structure and numeration of atoms in the cortisol molecule. (b) IR spectrum of a bare Au nanoantenna array and cortisol deposited from the solution with concentration $10 \mathrm{ng} / \mu \mathrm{L}$ on the array. Inset shows the detailed fragmentary IR spectrum of $40 \mathrm{ng}$ of cortisol after subtraction of the envelope line. 
under the influence of localized plasmonic enhancements, thus increasing the sensitivity limit. As a proposed further application of SEIRA, it was also shown that the detection limit of cortisol using SEIRA corresponds to $40 \mathrm{ng}$ - this opens up the possibility for determination of steroid concentrations in real biological assays.

\section{Experimental}

The uniform periodic arrays of linear Au nanoantennas with length 900 and $1900 \mathrm{~nm}$ and period of $5 \mu \mathrm{m}$ were fabricated on (001)-oriented Si substrates by a direct writing nanolithographic machine (Raith-150, Raith GmbH, Germany) and covers an area of $3 \times 3 \mathrm{~mm}^{2}$, providing the LSPR band energies in the range from $1580-800 \mathrm{~cm}^{-1}$. To avoid interference in the SEIRA spectra, $400 \mu \mathrm{m}$ thick Si substrates were used. The fabrication process of nanoantenna arrays is very similar to that described in [24] for Au nanocluster arrays.

The structural parameters of nanoantenna arrays were controlled by a scanning electron microscopy (SEM) using the same Raith-150 system at $10 \mathrm{kV}$ acceleration voltage, $30 \mu \mathrm{m}$ aperture, and $6 \mathrm{~mm}$ working distance.

Ultrathin CoPc films with thickness 3 and $10 \mathrm{~nm}$ were formed using organic molecular beam vapor deposition onto arrays of $\mathrm{Au}$ nanoantennas. The thermal evaporation of the organic molecule was performed in a vacuum chamber at a pressure of $\approx 5 \times 10^{-8}$ mbar. The evaporation temperature was approximately $400{ }^{\circ} \mathrm{C}$ and the deposition rate was $\approx 0.5 \mathrm{~nm} / \mathrm{min}$. During the organic film growth, the substrate was kept at room temperature.

Cortisol (11ß)-11,17,21-trihydroxypregn-4-ene-3,20-dione) was purchased from Calbiochem (USA) and was used without further purification. Cortisol dissolved in ethanol at concentration of $10 \mathrm{ng} / \mathrm{mL}(27.6 \mu \mathrm{M})$ was deposited onto arrays of $\mathrm{Au}$ nanoantennas by drop-casting. The drop volume was $2 \mu \mathrm{L}$.

The LSPR energy on Au nanoantenna arrays with and without organic material was determined from the IR transmission measurements carried out by using a Bruker Vertex 80v Fourier transform infrared spectrometer in the spectral range of $600-4000 \mathrm{~cm}^{-1}$. The spectral resolution was $2 \mathrm{~cm}^{-1}$ over the whole spectra range. The ratio of the transmission spectra polariszed along and perpendicular to the long axis of the bare nanoantennas and with deposited organic films was analyzed. The noise level was below $0.1 \%$ in the IR experiments. The measurements were carried out at room temperature.

Non-polarized Raman spectra were measured using Labram spectrometers equipped with a Renishaw InVia Raman micro- scope (the laser beam was focused to a spot with a diameter of about $1 \mu \mathrm{m}$ ) in a backscattering geometry at room temperature. A HeNe laser was used as an excitation source at the wavelength of $632.8 \mathrm{~nm}(2.41 \mathrm{eV})$. A laser power of less than $100 \mu \mathrm{W}$ (before the microscope) was used to avoid possible effects of local heating. The spectral resolution was below $2.5 \mathrm{~cm}^{-1}$ over the whole spectral range.

\section{Acknowledgements}

The authors gratefully acknowledge financial support from Volkswagen Foundation, TMS project (TU Chemnitz), Russian Science Foundation (project n.14-12-01037) in part of simulation and fabrication of Au nanoantenna arrays and SEIRA measurements, and the Ministry of Education and Science of the Russian Federation. The authors are thankful to L.L. Sveshnikova and A.S. Medvedev for technical assistance.

\section{References}

1. Wang, L.; Yoon, M.-H.; Lu, G.; Yang, Y.; Facchetti, A.; Marks, T. J. Nat. Mater. 2006, 5, 893-900. doi:10.1038/nmat1755

2. Zhang, C.; Chen, P.; Hu, W. Chem. Soc. Rev. 2015, 44, 2087-2107. doi:10.1039/C4CS00326H

3. Dediu, V. A.; Hueso, L. E.; Bergenti, I.; Taliani, C. Nat. Mater. 2009, 8, 707-716. doi:10.1038/nmat2510

4. Gu, H.; Zhang, X.; Wei, H.; Huang, Y.; Wei, S.; Guo, Z. Chem. Soc. Rev. 2013, 42, 5907-5943. doi:10.1039/c3cs60074b

5. Robaschik, P.; Siles, P. F.; Bülz, D.; Richter, P.; Monecke, M.; Fronk, M.; Klyatskaya, S.; Grimm, D.; Schmidt, O. G.; Ruben, M.; Zahn, D. R. T.; Salvan, G. Beilstein J. Nanotechnol. 2014, 5, 2070-2078. doi:10.3762/bjnano.5.215

6. Barraud, C.; Bouzehouane, K.; Deranlot, C.; Kim, D. J.; Rakshit, R.; Shi, S.; Arabski, J.; Bowen, M.; Beaurepaire, E.; Boukari, S.; Seneor, P.; Mattana, R. Dalton Trans. 2016, 45, 16694-16699. doi:10.1039/C6DT02467J

7. Lach, S.; Altenhof, A.; Tarafder, K.; Schmitt, F.; Ali, M. E.; Vogel, M.; Sauther, J.; Oppeneer, P. M.; Ziegler, C. Adv. Funct. Mater. 2012, 22 , 989-997. doi:10.1002/adfm.201102297

8. Serri, M.; Wu, W.; Fleet, L. R.; Harrison, N. M.; Hirjibehedin, C. F.; Kay, C. W. M.; Fisher, A. J.; Aeppli, G.; Heutz, S. Nat. Commun. 2014, 5, No. 3079. doi:10.1038/ncomms4079

9. Schmaus, S.; Bagrets, A.; Nahas, Y.; Yamada, T. K.; Bork, A.; Bowen, M.; Beaurepaire, E.; Evers, F.; Wulfhekel, W. Nat. Nanotechnol. 2011, 6, 185-189. doi:10.1038/nnano.2011.11

10. Annese, E.; Fujii, J.; Vobornik, I.; Panaccione, G.; Rossi, G. Phys. Rev. B 2011, 84, 174443. doi:10.1103/PhysRevB.84.174443

11. Larson, T. A.; Bankson, J.; Aaron, J.; Sokolov, K. Nanotechnology 2007, 18, 325101. doi:10.1088/0957-4484/18/32/325101

12. de la Chapelle, M. L.; Pucci, A., Eds. Nanoantenna: Plasmon-Enhanced Spectroscopies for Biotechnological Applications; Pan Stanford Publishing Pte. Ltd.: Singapore, 2013. doi:10.1201/b14594

13. Aroca, R. Surface-enhanced Vibrational Spectroscopy; John Wiley \& Sons: New Jersey, U.S.A., 2006. doi:10.1002/9780470035641

14. Steinle, T.; Neubrech, F.; Steinmann, A.; Yin, X.; Giessen, H. Opt. Express 2015, 23, 11105-11113. doi:10.1364/OE.23.011105 
15. Schatz, G. C.; Young, M. A.; Van Duyne, R. P. Electromagnetic Mechanism of SERS. In Surface-Enhanced Raman Scattering: Physics and Applications; Kneipp, K.; Moskovits, M.; Kneipp, H., Eds.; Topics in Applied Physics, Vol. 103; Springer: Berlin, Germany, 2006; pp 19-45. doi:10.1007/3-540-33567-6_2

16. Neubrech, F.; Pucci, A.; Cornelius, T. W.; Karim, S.; García-Etxarri, A.; Aizpurua, J. Phys. Rev. Lett. 2008, 101, 157403. doi:10.1103/PhysRevLett.101.157403

17. Huck, C.; Neubrech, F.; Vogt, J.; Toma, A.; Gerbert, D.; Katzmann, J.; Härtling, T.; Pucci, A. ACS Nano 2014, 8, 4908-4914. doi:10.1021/nn500903v

18. Moskovits, M. Rev. Mod. Phys. 1985, 57, 783-826. doi:10.1103/RevModPhys.57.783

19. Kneipp, K.; Wang, Y.; Kneipp, H.; Perelman, L. T.; Itzkan, I.; Dasari, R. R.; Feld, M. S. Phys. Rev. Lett. 1997, 78, 1667-1670. doi:10.1103/PhysRevLett.78.1667

20. Adato, R.; Yanik, A. A.; Amsden, J. J.; Kaplan, D. L.; Omenetto, F.; Hong, M. K.; Erramilli, S.; Altug, H. Proc. Natl. Acad. Sci. U. S. A. 2009, 106, 19227-19232. doi:10.1073/pnas.0907459106

21. Lee, D.-K.; Kang, J.-H.; Lee, J.-S.; Kim, H.-S.; Kim, C.; Kim, J. H.; Lee, T.; Son, J.-H.; Park, Q.-H.; Seo, M. Sci. Rep. 2015, 5, 15459. doi:10.1038/srep15459

22. Lee, D.-K.; Kim, G.; Son, J.-H.; Seo, M. Proc. SPIE 2016, 9747 , 97470S. doi:10.1117/12.2212055

23. Aroca, R.; Pieczonka, N.; Kam, A. P. J. Porphyrins Phthalocyanines 2001, 5, 25-32. doi:10.1002/1099-1409(200101)5:1<25::AID-JPP301>3.0.CO;2-A

24. Milekhin, A. G.; Yeryukov, N. A.; Sveshnikova, L. L.; Duda, T. A.; Rodyakina, E. E.; Sheremet, E. S.; Ludemann, M.; Gordan, O. D.; Latyshev, A. V.; Zahn, D. R. T. Thin Solid Films 2013, 543, 35-40. doi:10.1016/j.tsf.2013.03.070

25. Oraevsky, A. A.; Oraevsky, A. N. Quantum Electron. 2002, 32, 79-82. doi:10.1070/QE2002v032n01ABEH002131

26. Sidorov, A. N. Opt. Spectrosc. 1976, 40, 280-283.

27. Meshkova, G. N.; Vartanyan, A. T.; Sidorov, A. N. Opt. Spectrosc. 1977, 43, 151-154.

28. El-Nahass, M. M.; Abd-El-Rahman, K. F.; Darwish, A. A. A. Mater. Chem. Phys. 2005, 92, 185-189. doi:10.1016/j.matchemphys.2005.01.008

29. Zeman, E. J.; Carron, K. T.; Schatz, G. C.; Van Duyne, R. P. J. Chem. Phys. 1987, 87, 4189-4200. doi:10.1063/1.452923

30. Kötz, R.; Yeager, E. J. Electroanal. Chem. Interfacial Electrochem. 1980, 113, 113-125. doi:10.1016/S0022-0728(80)80515-8

31. Toman, P.; Nešpůrek, S.; Yakushi, K. J. Porphyrins Phthalocyanines 2002, 6, 556-562. doi:10.1142/S1088424602000695

32. Milekhin, I. A.; Kuznetsov, S. A.; Rodyakina, E. E.; Milekhin, A. G.; Latyshev, A. V.; Zahn, D. R. T. Beilstein J. Nanotechnol. 2016, 7, 1519-1526. doi:10.3762/bjnano.7.145

33. Neubrech, F.; Weber, D.; Enders, D.; Nagao, T.; Pucci, A. J. Phys. Chem. C 2010, 114, 7299-7301. doi:10.1021/jp908921y

34. Toma, A.; Tuccio, S.; Prato, M.; De Donato, F.; Perucchi, A.; Di Pietro, P.; Marras, S.; Liberale, C.; Zaccaria, R. P.; De Angelis, F.; Manna, L.; Lupi, S.; Di Fabrizio, E.; Razzari, L. Nano Lett. 2015, 15, 386-391. doi:10.1021/nl503705w

35. D'Andrea, C.; Bochterle, J.; Toma, A.; Huck, C.; Neubrech, F.; Messina, E.; Fazio, B.; Maragò, O. M.; Di Fabrizio, E.; de La Chapelle, M. L.; Gucciardi, P. G.; Pucci, A. ACS Nano 2013, 7, 3522-3531. doi:10.1021/nn4004764

36. Cherkasova, O. P. Biochemistry (Moscow) 2007, 1, 172-175. doi:10.1134/S1990750807020126
37. Holder, G. Measurement of Glucocorticoids in Biological Fluids. In Hormone Assays in Biological Fluids; Wheeler, M. J.; Hutchinson, J. S. M., Eds.; Methods in Molecular Biology, Vol. 324; Humana Press: Totowa, NJ, U.S.A.; pp 141-157. doi:10.1385/1-59259-986-9:141

38. Cherkasova, O. P.; Selyatitskaya, V. G. Biochemistry (Moscow) 2013, 7, 90-94. doi:10.1134/S1990750813010034

39. Al Sharef, O.; Feely, J.; Kavanagh, P. V.; Scott, K. R.; Sharma, S. C. Biomed. Chromatogr. 2007, 21, 1201-1206. doi:10.1002/bmc.877

40. Lee, S.; Lim, H.-S.; Shin, H.-J.; Kim, S.-A.; Park, J.; Kim, H.-C.; Kim, H.; Kim, H. J.; Kim, Y.-T.; Lee, K.-R.; Kim, Y.-J. J. Anal. Methods Chem. 2014, 2014, 787483. doi:10.1155/2014/787483

41. Mitchell, J. S.; Lowe, T. E.; Ingram, J. R. Analyst 2009, 134, 380-386. doi:10.1039/B817083P

42. Kasal, A.; Budesinsky, M.; Griffiths, W. J. Spectroscopic Methods of Steroid Analysis. In Steroid Analysis; Makin, H. L. J.; Gower, D. B., Eds.; Springer: Berlin, Germany, 2010; pp 27-161. doi:10.1023/b135931_2

43. Spectral Database for Organic Compounds (SDBS), http://sdbs.db.aist.go.jp/sdbs/cgi-bin/direct_frame_top.cgi, SDBS No. 10001, hydrocortisone.

44. Rojek, B.; Wesolowski, M. J. Therm. Anal. Calorim. 2017, 127, 543-553. doi:10.1007/s10973-016-5441-7

\section{License and Terms}

This is an Open Access article under the terms of the Creative Commons Attribution License (http://creativecommons.org/licenses/by/4.0), which permits unrestricted use, distribution, and reproduction in any medium, provided the original work is properly cited.

The license is subject to the Beilstein Journal of

Nanotechnology terms and conditions:

(http://www.beilstein-journals.org/bjnano)

The definitive version of this article is the electronic one which can be found at: $\underline{\text { doi:10.3762/bjnano.8.99 }}$ 